\title{
Effect of Pigment Concentration on Mechanical Properties of Polycarbonate
}

\author{
Vaclav Janostik ${ }^{1, *}$ and Vojtech Senkerik ${ }^{1}$ \\ 1 Tomas Bata University in Zlin, TGM 5555, 76001 Zlin, Czech Republic
}

\begin{abstract}
This experimental study deals with the influence of pigments on the mechanical properties of polymeric polycarbonate material Lexan $923 \mathrm{c}$, which is commonly used in industry and is being processed with injection technology. This material was stained with three different pigments from Lifokolor. It was also stained with 6 different concentrations of selected pigments. Emphasis was placed on measuring the tensile strength, impact strength and hardness of polyurethane mixtures.
\end{abstract}

\section{Introduction}

Nowadays polymeric materials are irreplaceable in many industries. They are exclusive material for their mechanical properties and shaping capabilities. To obtain higher effectivity of production, a number of complicated parts is made by injection technology. Often, customer requirements are too high. Above all, the biggest problem is adhering to the desired color range and the mechanical properties of the part. Therefore, compromise between materials and pigments should be found.

There are several basic color methods. However, these methods are associated with the final process. We are focusing on the injection process, which has a high ability to reproduce complicated parts.

The granulate for injection can be colored in a dry manner. This is a direct mixing of the natural granulate with the pigments in the mixing device. Thereafter, this prepared mixture is transferred in the hopper of the injection moulding machine. The distribution of the colored concentrate of this mixture is not perfect. Even further strained and sedimentation of the colored concentrate is observed due to the shock of the injection unit. Therefore, this method is suitable for products with lower demands on color accuracy. To obtain more precise distribution of color concentrate, bulk or mass dispensers are used.

The colored concentrate consists of a polymeric carrier which is miscible with the polymer. In the polymeric carrier, the pigment is incorporated up to $90 \%$. The final colour effect must be consist with the customer's requirements. The way of dosing and mixing of the coloured concentrate is important for desired values of the colour shade. The way of dosing is also very important for the final mechanical properties of the part.

\section{Methods}

\subsection{Type of polymeric material and pigment} Lexan 923 polycarbonate (PC) from SABIC was selected for studying of mechanical properties. This material is characterized by high impact resistance, it is strong and flexible. Excludes the possibility of cracks. In contact with fire, products become soft and melt, fire is not support and it is not expand. Low weight with good mechanical properties guarantee a long service life. The test specimens were made by injecting this polymeric material with a different concentration of three kinds of pigment colors.

For the coloring of the natural polymeric material, three color pigment types have been used, which are commonly used in industry. These pigments were purchased from LIFOCOLOR s.r.o. in basic color shades. This pigment is stable up to $290^{\circ} \mathrm{C}$

\subsection{Formation of the mixture}

Dry blending was used for preparing of the mixture. Stirring was always carried out in the exact ratio of Lexan 923 polymer granulate with a certain type of pigment. Mixing systems commonly used in industry were used. The mixtures were mixed in concentrations (1-6)\%; total weight of the final mix was $1500 \mathrm{~g}$. The precision laboratory height of the HELAGO HL - 2000i was used for weighing.

Table 1. Formation of the mixture.

\begin{tabular}{|c|c|c|c|c|}
\hline$C_{\text {pigment }}$ & $\mathrm{C}_{\text {polymer }}$ & $\mathrm{m}_{\text {pigment }}$ & $\mathrm{m}_{\text {polymer }}$ & $\mathrm{M}_{\text {mix }}$ \\
\hline $1 \%$ & $99 \%$ & $15 \mathrm{~g}$ & $1485 \mathrm{~g}$ & $1500 \mathrm{~g}$ \\
\hline $2 \%$ & $98 \%$ & $30 \mathrm{~g}$ & $1470 \mathrm{~g}$ & $1500 \mathrm{~g}$ \\
\hline $3 \%$ & $97 \%$ & $45 \mathrm{~g}$ & $1455 \mathrm{~g}$ & $1500 \mathrm{~g}$ \\
\hline $4 \%$ & $96 \%$ & $60 \mathrm{~g}$ & $1440 \mathrm{~g}$ & $1500 \mathrm{~g}$ \\
\hline $5 \%$ & $95 \%$ & $75 \mathrm{~g}$ & $1425 \mathrm{~g}$ & $1500 \mathrm{~g}$ \\
\hline $6 \%$ & $94 \%$ & $90 \mathrm{~g}$ & $1410 \mathrm{~g}$ & $1500 \mathrm{~g}$ \\
\hline
\end{tabular}

\footnotetext{
* Corresponding author: vjanostik@utb.cz
} 


\subsection{Production of test specimens}

The test samples were created by injection molding technology on the ARBURG Allrounder 470H 1000-400 Injector. This machine can be optimally dimensioned for applications of all known injection technologies.

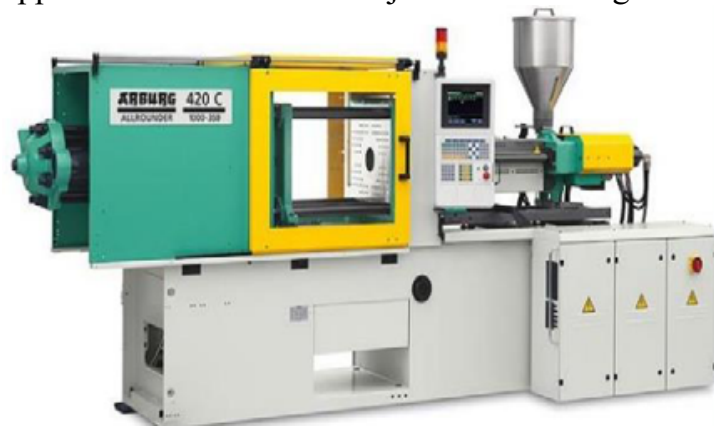

Fig 1. Injection machine

Two types of standardized test bodies were selected. The first body is designed for tensile testing according to CSN EN ISO 527-1 type 2 and the second body for impact strength is degined according to ČSN EN ISO $179-1$

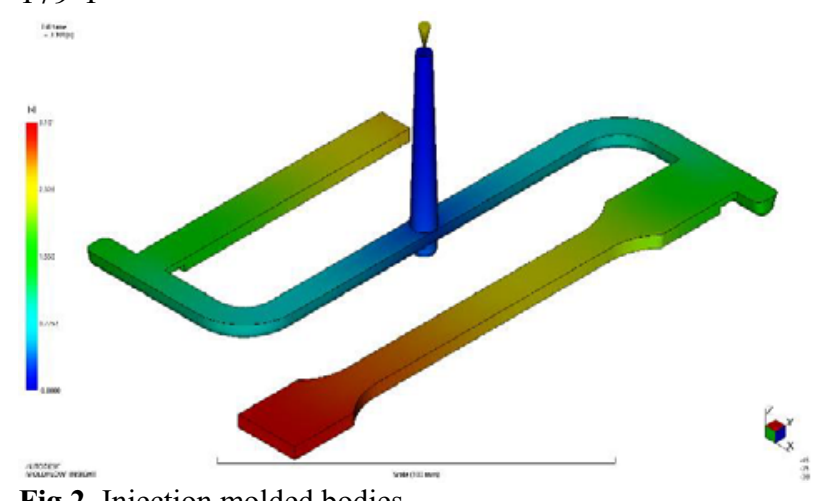

Fig 2. Injection molded bodies

Injection of test bodies was always carried out under the same technological conditions.

Table 2. Injection Parameters.

\begin{tabular}{|l|r|l|}
\hline mold temperature & 60 & ${ }^{\circ} \mathrm{C}$ \\
\hline plastication pach & 24 & $\mathrm{Mm}$ \\
\hline injection pressure & 800 & $\mathrm{Bar}$ \\
\hline touch pressure & 300 & $\mathrm{Bar}$ \\
\hline time touch pressure & 15,5 & $\mathrm{~S}$ \\
\hline injection speed & 40 & $\mathrm{~mm} / \mathrm{s}$ \\
\hline patch & 8 & $\mathrm{~mm}$ \\
\hline cool time & 30 & $\mathrm{~S}$ \\
\hline 1.zone temperature & 40 & ${ }^{\circ} \mathrm{C}$ \\
\hline 2. zone temperature & 270 & ${ }^{\circ} \mathrm{C}$ \\
\hline 3. zone temperature & 275 & ${ }^{\circ} \mathrm{C}$ \\
\hline 4. zone temperature & 280 & ${ }^{\circ} \mathrm{C}$ \\
\hline 5. zone temperature & 285 & ${ }^{\circ} \mathrm{C}$ \\
\hline nozzle temperature & 290 & ${ }^{\circ} \mathrm{C}$ \\
\hline
\end{tabular}

\section{Experiment}

Materials were tested by tensile test, flexural impact test, hardness test, and dimensional measurements to evaluate shrinkage. To compare the physical (mechanical) properties of polymer materials (PC) which was influenced by different pigment concentrations, a tensile test machine, charpy hammer, portal gauge and hardness hardness tester (Shore D) was used.

\subsection{Impact bending test}

The HIT impact hammer automatically detects the used pendulum. Therefore, HIT provides accurate measurement data within the specified range and corresponding to the standard. A double carbon rod was used to make the pendulums. High impact strength and high mass concentration at the point of impact are achieved. The impact hammer has an optional pendulum brake. The electronics include a high-resolution digital sensor for accurate angular swing measurement. Each set of measurements was performed on 20 test bodies

Fig 3. Impact bending test

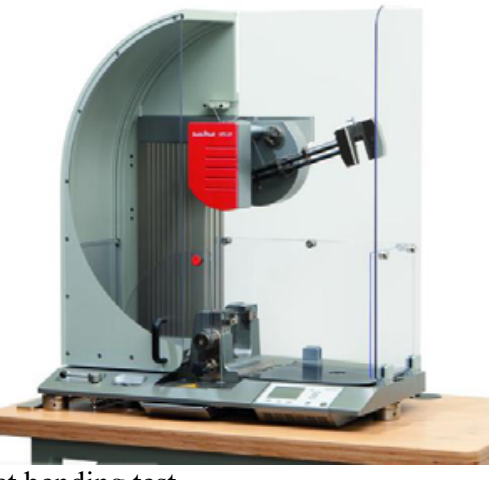

\subsection{Tensile stress machine}

After producing of the testing samples a test was carried out to determine the tensile stress-strain properties and also the test to determine the tear strength. In both cases the testing samples were clamped into jaws at both ends in the tensile stress machine Tensometer 2000 by Alpha Technologies. (Fig. 5). Test sample dumbbell was stretched by the prescribed constant speed $50 \mathrm{~mm} / \mathrm{min}$ until they were torn. In case of test sample crescent, graves and trouser, stretching speed was $100 \mathrm{~mm} / \mathrm{min}$.

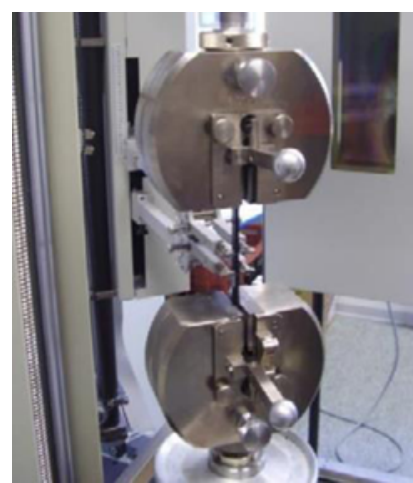

Fig 4. Tensile stress experiment 


\section{Results}

\subsection{Bending impact test}

The bending impact test was measured according to EN ISO 179-1. The test bodies were set up horizontally on the supports and hammered by impact hammer strikes. The test bodies were $80 \times 4 \times 10$ and were fitted with a notch type $\mathrm{V}$ at a depth of $2 \mathrm{~mm}$. Twenty test bodies were used for each set of measurements.

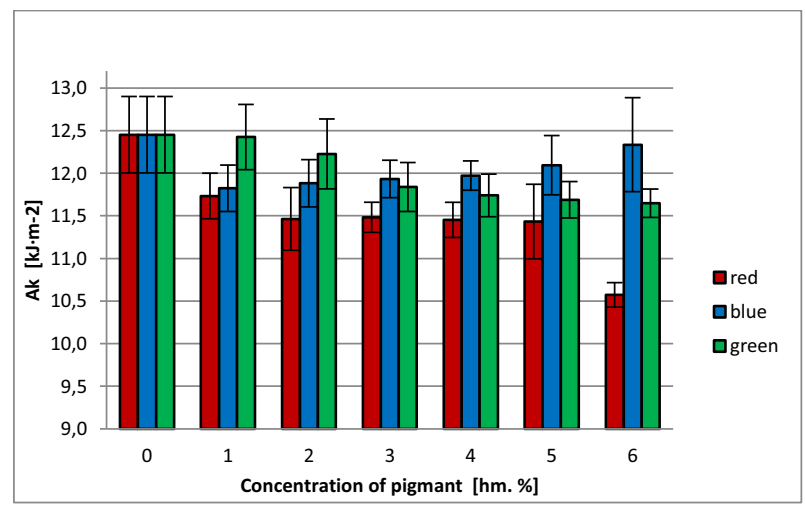

Fig 5. Noch tougness

The bending impact test determines the stiffness of the test body. When adding a red (Lifocolor-CR-Red 3620 F/PC) (red) or green (Greocolor-CR-Green 4842 F/PC) natural green polymer pigment (lexan 923) a decrease in notched toughness can be observed with increasing pigment concentration. On the other hand, the addition of blue (Lifocolor -CR-Blue 50009 F / PC) (blue) pigment increased the notch toughness. It has also been shown that by adding the pigments, the toughness was reduced in comparison with the natural material.

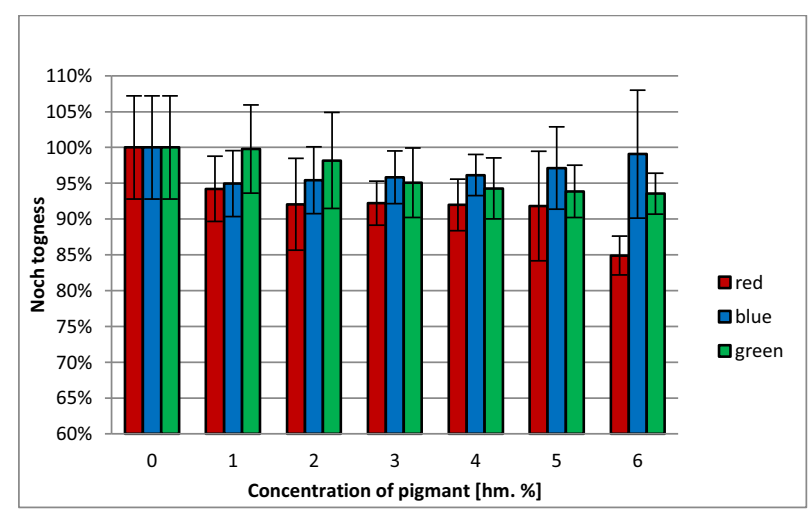

Fig 6. Proportional graph of notch toughness

At $1 \%$ concentration of the red pigment there was a $6 \%$ decrease in notch toughness. Also a negative trend can be seen. As the concentration of red pigment increases, the impact strength decreases. At $6 \%$ concentration of the red pigment, the toughness decreases by up to $15 \%$

At $1 \%$ concentration of the green pigment, the impact strength value is the same as the value of the transparent material. At $6 \%$ concentration there was a $7 \%$ decrease in impact strength. The green pigment creates a more stable mixture with a decrease in the impact strength of the transparent material, and the stiffness of the test body has decreased.

At $1 \%$ concentration of the blue pigment, the toughness dropped by $5 \%$. Further, the increasing trend is observed, at $6 \%$ concentration, the notched toughness value approaches the value of the transparent material. There was a $1 \%$ drop on the reference sample.

\subsection{Tension test}

The tensile test was performed according to the standard CSN EN ISO527-1 on the ZWICK Roell 1456 Testing Machine. The test bodies were shaped in the form of blades - type II. Twenty test specimens were used for each set of measurements. The temperature in the test room was $22^{\circ} \mathrm{C}$.

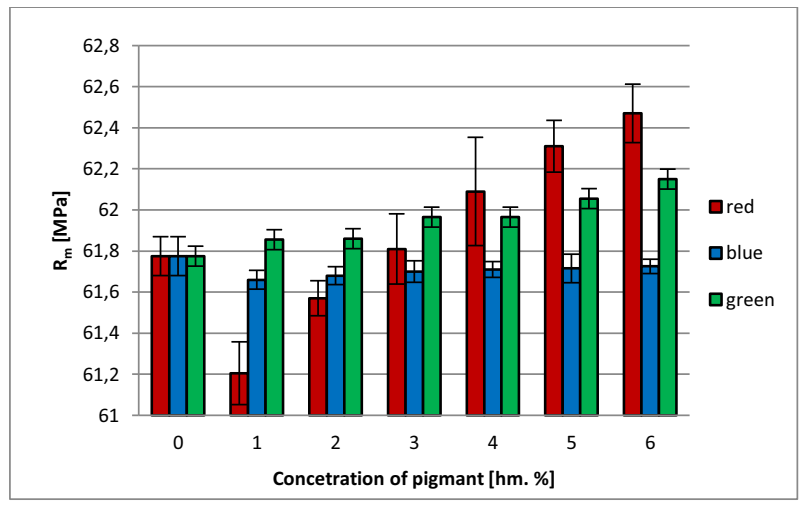

Fig 7. Limit of strength

\section{$249 / 5000$}

The tensile test indicates the strength of the material. When the red or green pigment was added to the natural polymer (lexan 923), the tensile strength was increased. On the other hand, when the blue pigment was added, a slight decrease in the tensile strength is observed.

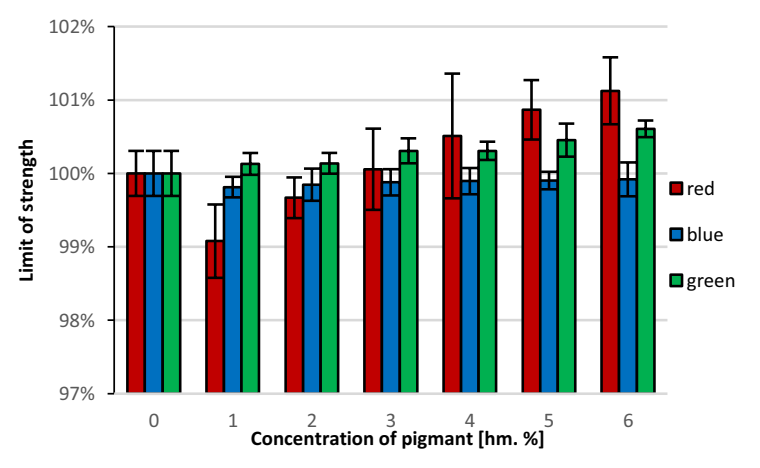

Fig 8. Proportional graph Limit of strength

At $1 \%$ concentration of the red pigment, the strength limit was decreased by $1 \%$, where a growing tren is evident. At $6 \%$ pigment concentration, the $1 \%$ strength limit was increased.

At $1 \%$ concentration of the green pigment, the strength limit was slightly increased. The $6 \%$ concentration increased by $0.6 \%$.

In the blue pigment, the tensile strength of the tensile strength dropped. This value fluctuated within a range of $0.5 \%$. 


\subsection{Shore $D$}

The hardness test (Shore D) was performed according to ISO 7619-1 standard on the Omag Affri ART 13 hardness tester. The test bodies had a thickness at the test site of $8 \mathrm{~mm}$ and their surface was smooth and clean. Ten test bodies were used for each set of measurements and 10 measurements were performed. The temperature in the test room was $22^{\circ} \mathrm{C}$.

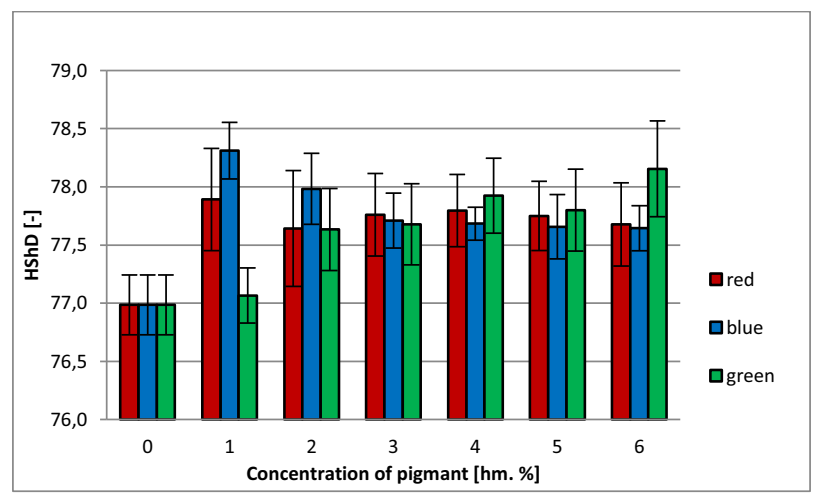

Fig 9. Shore D

Hardness results (Shore D) show that when a red pigment was added to the PC material (Lexan 923), the hardness increased and the trend was decreasing with increasing concentrations. A similar phenomenon occurred with the use of a blue pigment. On the other hand, the green pigment showed a growing trend.

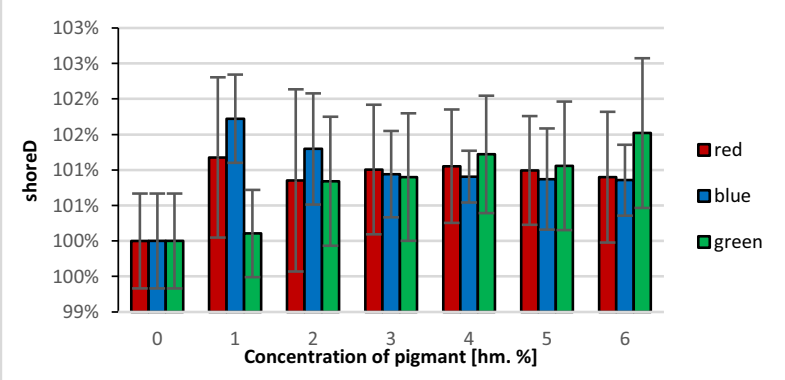

Fig 10. Proportional graph ShoreD

The change of the hardness is the most notably with $1 \%$ blue pigment concentration. When concentration grew by $2 \%$, the trend slightly declining. A similar phenomenon occurs when the red pigment is used. At $1 \%$ concentration the hardness increased by $1 \%$. However, with the increasing concentration of the pigment, there was a slight decrease in hardness. The intense phenomenon occurred with the green pigment. At $1 \%$ concentration there was a slight increase in hardness. He was also watching the growing trend. At a concentration of $6 \%$, the hardness increased by $2 \%$ with a transparent material

\section{Conclusion}

Impact strength evaluation shows that the type of pigment selected significantly influences the stiffness of the body. When red pigments were used at higher concentrations, the stiffness decreased up to $15 \%$. The blue pigment influenced the stiffness of the material to a minimum.
The tensile test evaluation shows that pigments do not significantly affect the tensile strength. Selection of the right concentration will result in minimal deviations from the strength limit.

Hardness is also influenced but only on a small scale. We are in the area of minimum deviations when a $3 \%$ pigment concentration is used. At this concentration, the materials have very similar mechanical properties.

\section{Acknowledgement}

This paper is supported by the internal grant of TBU in Zlin No. IGA/FT/2017/010 funded from the resources of specific university research and by the Ministry of Education, Youth and Sports of the Czech Republic within the National Sustainability Programme project No. LO1303 (MSMT-7778/2014) and also by the European Regional Development Fund under the project CEBIA-Tech No. CZ.1.05/2.1.00/03.0089

\section{References}

1. M. Ovsik, D. Manas, M. Manas, M. Stanek, M. Hribova, K. Kocman, D. Samek, Irradiated Polypropylene Studied by Microhardness and WAXS, Chemicke listy 106, (2012)

2. M. Stanek, M. Manas, D. Manas, V. Pata, S. Sanda, V. Senkerik, A. Skrobak, How the Filler Influence the Fluidity of Polymer, Chemicke listy, (2011)

3. M. Stanek, D. Manas, M. Manas, O. Suba, Optimization of injection molding process, Intl. J. of Math. and Computers in Simul., Vol.5, No.5, (2011)

4. J. Javorik, M. Stanek, The shape optimization of the pneumatic valve diaphragms, Intl. J. of Math. and Computers in Simul., Vol.5, No.4, (2011)

5. M. Stanek, D. Manas, M. Manas, J. Javorik, Simulation of injection molding process by cadmould rubber, Intl. J. of Math. and Computers in Simul., Vol.5, No.5, (2011)

6. M. Ovsik, D. Manas, M. Manas, M. Stanek, M. Bednarik, P. Kratky, Effect of beta irradiation on microhardness of polyamide 6, Key Engineering Materials, vol. 586, (2014)

7. M. Manas, D. Manas, M. Stanek, A. Mizera, M. Ovsik, Modification of polymer properties by irradiation properties of thermoplastic elastomer after radiation cross-linking, Asian Journal of Chemistry, vol. 25, Issue 9, (2013)

8. Tim A. Osswald, International Plastics Handbook: the Resource for Plastics Engineers (fourth ed.) Hanser Publishers, Munich (2006)

9. Dobransky, J., et al. Comparison of Cooling Variants by Simulation Software. Advanced Materials Research. Vol. 801 (2013). pp. 75-80. ISSN 1022-6680.

10. Behalek, L. and Dobránsky, J. Conformal cooling of the injection moulds. Applied Mechanics and Materials. Vol. 308 (2013). pp. 127-132. ISSN 1660-9336. 